\title{
Leveraging RF Power for Intelligent Tag Networks
}

\author{
Emre Salman \\ Stony Brook University \\ Stony Brook, NY 11794, USA \\ emre.salman@stonybrook.edu \\ Samir Das \\ Stony Brook University \\ Stony Brook, NY 11794, USA \\ samir.das@stonybrook.edu
}

\begin{abstract}
A novel framework and related methodologies are described to leverage RF power for building intelligent and battery-free devices with communication and computation capabilities. These passive devices are envisioned to make significant impact for the popular vision of smart dust due to extreme low power operation. The communication framework relies on tag-to-tag backscattering with very limited energy resources. The computing framework relies on a novel AC computing methodology that facilitates local data processing with an order of magnitude less power consumption. These enabling technologies, as described in this paper, revitalize the concept of smart dust with significant impact on various application domains such as smart spaces, implantable devices, and environmental/structural monitoring.
\end{abstract}

\section{KEYWORDS}

backscatter-based communication; RFID; RF tags; power harvesting; RF power; wireless energy; adaptive power management; wireless sensing; AC computing; decision making

\section{ACM Reference Format:}

Emre Salman, Milutin Stanaćević, Samir Das, and Petar M. Djurić. 2018. Leveraging RF Power for Intelligent Tag Networks. In GLSVLSI '18: 2018 Great Lakes Symposium on VLSI, May 23-25, 2018, Chicago, IL, USA. ACM, New York, NY, USA, 6 pages. https://doi.org/10.1145/3194554.3194621

\section{INTRODUCTION}

The powerful vision of smart dust, proposed in the late 1990s, consists of tiny mote-like devices that are distributed throughout an environment and possess critical capabilities such as sensing, computation, and wireless communication [13]. Despite the significant impact this vision had in the field of wireless sensor networks, the original anticipated framework has not yet come true since delivering power to each of these devices has been a primary barrier. Existing battery technologies are not only highly costly, but also impractical since it would require hundreds of millions of battery

Permission to make digital or hard copies of part or all of this work for personal or classroom use is granted without fee provided that copies are not made or distributed for profit or commercial advantage and that copies bear this notice and the full citation on the first page. Copyrights for third-party components of this work must be honored.

For all other uses, contact the owner/author(s).

GLSVLSI '18, May 23-25, 2018, Chicago, IL, USA

(C) 2018 Copyright held by the owner/author(s).

ACM ISBN 978-1-4503-5724-1/18/05

https://doi.org/10.1145/3194554.3194621

\author{
Milutin Stanaćević \\ Stony Brook University \\ Stony Brook, NY 11794, USA \\ milutin.stanacevic@stonybrook.edu
}

\author{
Petar M. Djurić \\ Stony Brook University \\ Stony Brook, NY 11794, USA \\ petar.djuric@stonybrook.edu
}

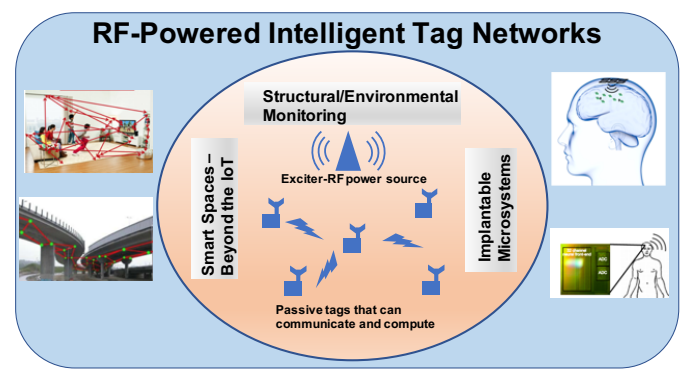

Figure 1: RF-powered intelligent tags that can communicate with each other through backscattering and achieve local computation through efficient AC computing methodology.

changes a day. Furthermore, limited power densities of conventional electrochemical charge storage techniques place stringent constraints on form factor.

In this paper, tiny tags that are powered by harvesting available RF energy are introduced, as conceptually illustrated in Fig. 1. RF power can be provided both from ambient sources such as cellular, TV or WiFi signals as well as from dedicated wireless power sources such as RF exciters. Dedicated RF power sources have been successful in providing the required energy for radio frequency identification (RFID) and wireless charging applications.

Contrary to traditional approaches that are based on active radios, the communication among the tags is achieved by backscattering principle where the data are transmitted by reflecting an incident RF signal while modulating the input impedance of the tag antenna. The receiving tag decodes the transmitted signal through passive and low power envelope detection. Furthermore, the backscattering principle is exploited to monitor the wireless channel and therefore carry out wireless sensing, where the tags can detect new object in proximity. This requires energy-efficient local processing and decision making, which is achieved by the proposed AC computing methodology. Contrary to conventional methods that rely on rectifying the harvested AC signal and conventional DC-based computing, in the proposed method, the local processing core directly uses the harvested AC signal by leveraging adiabatic circuit theory. Thus, the significant losses related to rectification are eliminated and the computing is performed significantly more efficiently due to adiabatic charging and charge-recycling. The proposed passive tags have enormous implications both for (a) near-field (range limited) applications such as brain implantable devices for neural recording and stimulation and (b) for far-field applications such as smart spaces. 
The rest of the paper is organized as follows. Related background material is provided in Section 2. Contributions of this work are highlighted in Section 3. The proposed tag architecture and critical components are detailed in Section 4. Several related applications where the proposed tags can be utilized are discussed in Section 5 . Finally, conclusions are offered in Section 6.

\section{BACKGROUND}

Conventional RF-powered tags utilize RF power harvesting and backscatter communication to deliver sufficient functionality to communicate small amounts of information. Backscattering is based on modulating an external RF signal incident on the antenna of the device. The modulation is achieved by modifying the impedance levels seen by the antenna, thereby changing its reflection coefficient. This requires very small amount of power which, with appropriate circuits, can be supplied by the external RF signal itself. Today, the most widely used embodiment of this technology is in RFIDs [10, 11]. In RFID, the external RF signal is provided via a relatively higher power embedded computer, possessing an active radio, referred to as the 'reader.' The reader also receives and interprets the modulated signal backscattered by the tag. RFID has long been standardized and is now widely deployed in logistics and inventory applications to perform identification and tracking [1]. Variations of RFID exist as research platforms such as tags with sensors $[9,47]$ and tags with programming/computational ability (Computational RFID) [32, 47].

Two recent innovations have improved the possibility of ubiquitous deployment of such tags. These include 1) use of ambient RF signals (e.g., TV or $\mathrm{WiFi}$ ) to provide the external RF signal and/or to power the tag $[17,21]$ and 2) tag-to-tag backscatter communications $[3,21,26]$. Use of ambient RF signals enables the tags to be deployed anywhere with no other infrastructure support necessary. Tag-to-tag backscatter implies that there is no need to have highpowered reader devices in the neighborhood to read the tag signals; the tags themselves can read and in turn relay the information using multihop routing. This enables highly scalable deployment.

\section{CONTRIBUTIONS}

This work benefits from existing tag-to-tag backscattering principle, but significantly enhances the basic technique for improved robustness and range. Furthermore, conventional tags suffer from lack of sufficient compute ability due to limited available power. Transmitting large amounts of raw data is not practical due to significant power cost and additional latency for the decision making process. Thus, an AC computing methodology is proposed to enhance the energy efficiency by more than an order of magnitude. Conventional digital logic in these applications typically relies on ultra-low voltage design with subthreshold operation [19]. Even though power levels in the range of nanowatts are achieved, an important limitation of these approaches is significant reduction in performance (clock frequency in range of several $\mathrm{kHz}$ ) which is critical for applications where real-time inference and decision making are required.

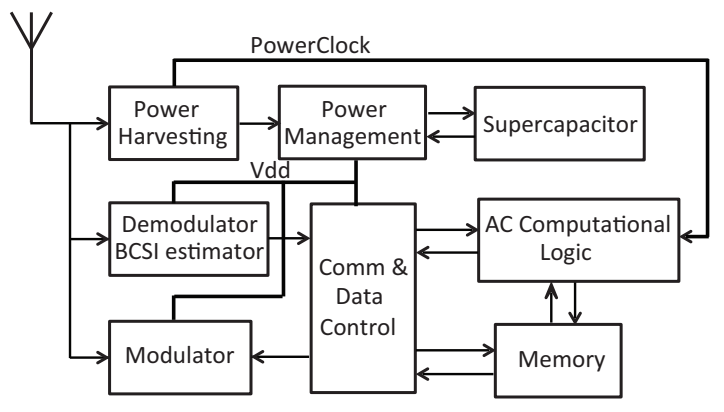

Figure 2: Proposed tag architecture depicting different modules.

\section{PROPOSED TAG ARCHITECTURE}

A high level block diagram of the proposed tag architecture, the principle building block of an intelligent tag network, is shown in Fig. 2. The architecture is similar to the traditional RFID tag with additional functionality and revised communication module optimized for tag-to-tag communication. The tag comprises antenna, $\mathrm{RF}$ energy harvesting and power management modules, modulator and demodulator for tag-to-tag communication, DC control and AC computational logic, along with memory and energy storage element, a supercapacitor, within a small form-factor.

A single frequency RF energy harvesting at $915 \mathrm{MHz}$ or at $2.4 \mathrm{GHz}$ is possible, as well as multi-band RF energy harvesting with more complex antenna design $[5,18,38]$. The incident RF signal is divided between sinusoidal power-clock signal required for the operation of AC logic, a DC supply voltage generated through AC-DC conversion for operation of DC logic and communication module, with extra energy being stored. The tags are designed to operate on a harvest-use-store principle with a supercapacitor as an energy storage element. Based on the incident RF power, stored energy and possible actions that can be taken, the logic implemented in the power management module directs the operation of the tag. The tag can integrate different ultra-low power stand-alone sensors. Additionally, the backscatter links themselves provide a form of sensing ability (sensing of backscatter channel state information(BCSI) integrated in demodulator) that can be exploited to perform network-wide inference. The computational logic, based on the collected data, is used for extracting information about the tag's environment.

\subsection{RF Energy Harvester}

The RF energy harvesting circuit comprises voltage multiplier that converts the incident AC signal to DC voltage as well as several signal conditioning blocks to obtain the required AC signals (powerclock) for AC computational logic. Different number of stages of the voltage multiplication can be used to boost the DC voltage. The energy efficiency, which is the amount of energy transfered by the voltage multiplier, in the conventional voltage multiplier is optimized for a certain small range of input powers. While the tag is listening for or receiving the message from another tag, the incident power at the antenna input is split between the energy harvester and the demodulator. In conventional RFID tags, due to a high modulation index of the received signal, the power ratio is determined solely by the optimization of the energy harvesting. 


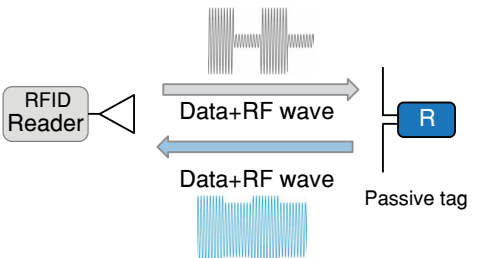

(a)

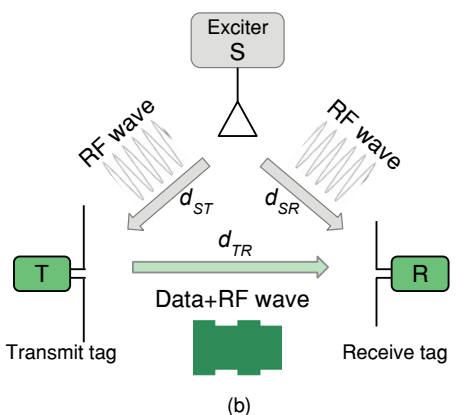

(b)

Figure 3: (a) General RFID communication setup, (b) Backscatter tag-to-tag communication network setup.

However, in the tag-to-tag link, the low modulation index not only adds complexity to the design of the demodulator itself, but also calls for the co-design of the energy harvester and demodulator. As an example of this optimization strategy, we examined the performance of the demodulator for different ratios of power division between the demodulator and power harvester. We demonstrated that there exists optimal power ratio that optimizes the energy transfer and voltage sensitivity of the demodulator circuit [2].

Design of the energy harvester with high energy efficiency over a wide input power range presents significant challenge. Structures like dual-path rectifier have been proposed in the literature [23, 24], however the range of the input powers over which the efficiency was optimized is narrow compared to the range of the incident powers at which the tag should operate. An energy-aware architecture of the energy harvester that will optimize the energy efficiency over a wide range of the incident power is a potential research direction.

\subsection{Tag-to-Tag Link Design}

The focus in the design of the communication module of the proposed tag is to achieve robust and reliable tag-to-tag communication link across a wide range of incident power. In conventional RFID reader-tag links, a tag demodulates a signal with a very high modulation index which greatly increases the robustness of the link, as illustrated in Fig. 3(a). The backscatter tag-to-tag link is illustrated in Fig. 3(b). The amplitude of the baseband signal depends on the input power at both the transmitting and receiving tags, along with the phase at which the transmitting tag backscatters due to the relative phase difference between the superimposing excitation and backscatter signals. The detailed analysis is presented in [14]. The tag-to-tag link is asymmetric as the tag closer to the RF source, when it backscatters, produces signal with high modulation index, while when it is receiving, the modulation index of the received signal is low due to high input power.
4.2.1 Modulator Design. The traditional backscatter modulator transmits data by switching between two different terminating impedances connected to the antenna. In tag-to-tag link, the amplitude of the baseband signal depends on the relationship between the relative phase difference between the superimposing RF source excitation signal and the signal backscattered from transmitting tag, and the phase of the terminating impedance at the transmitter tag. At specific distances between the tags, there exists a null in the response at the receiving tag, that is no distinction between the received signals for both states of the modulator of transmitting tag. We have analyzed this problem in detail and proposed a transmission scheme in which modulator transmits messages at two orthogonal transmitter phases [36]. If signal is canceled in one transmitter phase, the amplitude will be maximum in the other phase. We implemented the modulator that reflects the incident signal at a different phase using variable terminating impedance. This increases the robustness of the link by preventing nulls in the response at the receiving tag at specific distances between tags [36].

4.2.2 Demodulator Design. The signal reception using passive envelope detection $[7,16,30]$ has been widely explored in the context of backscatter systems. However, application to tag-to-tag communication system gives rise to unique challenges. While in the forward (reader to tag) link of traditional RFID systems, the incoming signal at the tag has a very large modulation index, in tagto-tag link, the Rx tag receives a much weaker backscatter signal from a Tx tag in the presence of a much stronger external excitation signal. This means that the modulation index of the incoming signal is at least an order of magnitude lower.

We use the envelope detector as the input circuit at the interface with antenna followed by the passive filter to improve signal-tonoise ratio (SNR), as in the conventional RFID tags $[6,7,15,40]$. If there would be preceding RF gain stage, the power consumption of the tag would exceed $50 \mu \mathrm{W}$ [4], which is much higher than the power available to RF energy harvesting tags. The envelope detector is implemented as voltage doubler. Since we want to resolve small modulation index, the envelope detector is followed by architecture comprising an amplifier with integrated band-pass filter followed by comparator. The integrated high-pass filtering removes the baseline from the modulated signal. The high-gain amplifier is implemented as low-noise, low-power folded cascoded amplifier.

The proposed demodulator architecture with voltage doubler followed by an amplifier with integrated high-pass filtering was simulated in $45 \mathrm{~nm}$ CMOS technology with supply voltage of $1.1 \mathrm{~V}$. Averaged gain of amplifier is 64 which is enough to detect very weak signal. To demonstrate how the incoming signal with low modulation index can be distinguished, the input power to the tag was held at $-28 \mathrm{dBm}$ with ASK modulation and data rate of $10 \mathrm{kbps}$. At this incident power, we are able to resolve the received backscatter signal with the modulation index as low as $0.6 \%$ [14]. The power consumption of the demodulator is $1.2 \mu \mathrm{W}$.

4.2.3 Prototype platform for tag-to-tag communication. We have fabricated a discrete prototype of RF backscattering tag, shown in Fig. 4. In the initial prototype, RF power harvesting is not implemented and the tag is powered by a CR 1620 coin cell battery. The prototype tag includes a single dipole antenna and uses a discrete component conventional architecture of the modulator and the 


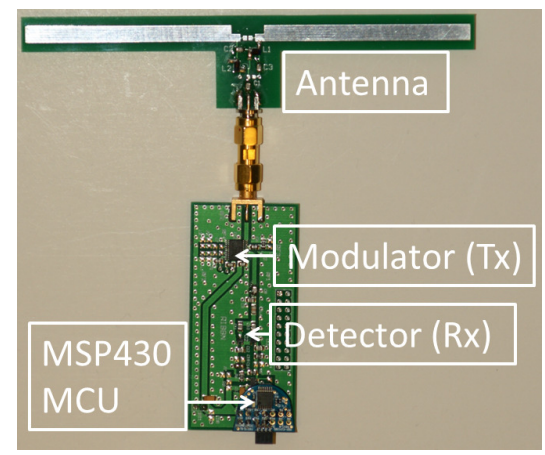

Figure 4: Prototype RF tag with dipole antenna and conventional modulator and demodulator implementation.

demodulator. In the demodulator, a two stage voltage multiplier, implemented using zero bias Schottky diode HSMS-285x series from Avago Technologies, is used for envelope extraction. To demonstrate the tag-to-tag link, we have performed experiments with dedicated RF source providing CW signal at $915 \mathrm{MHz}$ in lab environment. We have demonstrated that the maximum communication distance between tags at the incident power of $-20 \mathrm{dBm}$ at both tags is $3 \mathrm{~m}$ and that at the incident power of $-15 \mathrm{dBm}$ the range extends to $7 \mathrm{~m}$.

\subsection{Adaptive Power Management}

For the operation of a tag in a network of tags, in addition to the outlined challenges in the circuit design, the management of the operation of the tag on RF harvested energy and with a low capacity energy storage element introduces some unique challenges compared to the energy management of a traditional sensor node. Sensory nodes incorporate active radios that dominate the power budget, although significant steps have been made in reducing their power consumption $[4,8,12,27,31]$. The sensor nodes with energy harvesting have optimized energy-neutral operation that maximizes their performance with respect to the available energy, either on node level or application level [22, 25, 29, 33, 37, 39]. The balance between energy and performance is achieved through a wide range of adaptive techniques that control parameters like duty-cycle of the operation, transmit power of active radio and sensor reliability [20,35,41]. Backscatter-based communication in the proposed tag reduces the energy cost of the communication by a few orders of magnitude compared to the sensor node with active radio. This results in a different energy budget distribution for different modes of operation compared to the conventional sensor node and calls for different management techniques to be used in the tag's implementation.

In a network of tags, the power needed for backscattering at the Tx tags is small relative to the power needed to demodulate a signal. The power needed for demodulation again depends on the modulation index (lower power for higher modulation index) and is thus variable. The available RF power could be harvested in the supercapacitor on-tag (Fig. 2) that could be used if instantaneous power availability is limited for the function at hand (either transmit, receive or processing). However, due to the small size of the tag, the supercapacitor capacity is limited. Overall, the above issues lead to

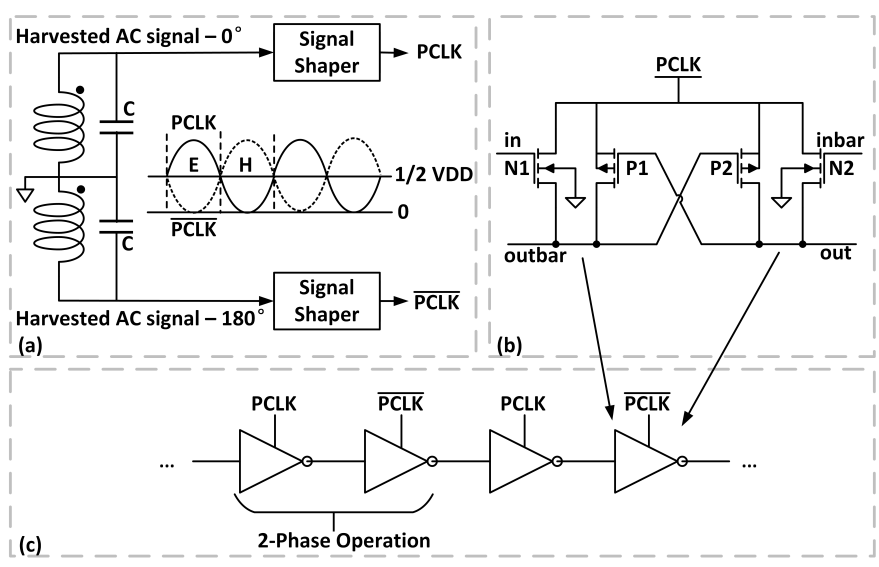

Figure 5: Adiabatic logic based AC computing approach: (a) 2-phase power-clock generation and signal shapers to eliminate negative voltage components, (b) schematic of a PAL based inverter, (c) chain of PAL logic gates powered with AC power-clock signals with $180^{\circ}$ phase difference.

an interesting power management plus network formation problem. Depending on the available RF power on a tag (this may depend on the exciter and tag locations and is different for different tags), the tag must decide on whether to demodulate a relatively weak backscatter signal from a neighbor. Due to the inherent redundancy in the network, the value of this action may not be significant relative to the needed energy. Thus, the network "links" that are to be used for communication are not well-defined even in a static network setting. The weaker links may only be occasionally used depending on the available RF power on the Rx tag and the value of using that link, By contrast, stronger links could be used more frequently as they may be less power constrained. A challenge here is the development of algorithms and protocols so that the links are used 'optimally' in the sense that the value of using a specific link for data routing is balanced with the energy budget of its $\mathrm{Rx}$ tag.

\subsection{On-Site AC Computing}

The proposed AC computing methodology enables energy-efficient data processing unit that can potentially be integrated within the passive tag [43]. AC computing leverages adiabatic circuit theory developed in the early 1990s [34, 46]. Extreme low power operation is achieved by eliminating rectification for logical operation and recycling charge during computation.

Existing adiabatic logic families should be reinvented for interoperability with the RF energy harvester described in Section 4.1. In this work, pass transistor adiabatic logic (PAL) [28] is used as an example, as as illustrated in Fig. 5 A PAL gate consists of two NMOS transistors N1, N2, and a pair of cross-coupled charging/recovering PMOS transistors P1, P2. The primary advantage of PAL is the ability to fully recycle charge since the NMOS transistors are connected to AC power supply. In addition, note that PAL is a two-phase logic where the AC supply of each consecutive gate is $180^{\circ}$ out-of-phase, as depicted in Fig. 5(c). Thus, when one of the gates is at the "evaluation" phase, the preceding gate is at the "hold" phase, maintaining the input signals stable for the evaluating gate. Note that the two inductors within the energy harvester are configured such that the 


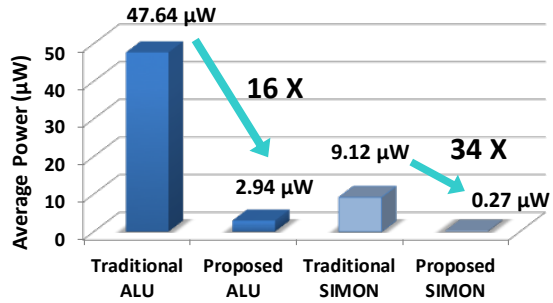

Figure 6: Comparison of power consumed by the 8-bit ALU and SIMON32/64 core, implemented using the proposed AC computing method and conventional method that relies on rectification and DC computing.

two harvested AC signals have $180^{\circ}$ phase difference. PAL, however, cannot correctly operate with the harvested AC signal that has both positive and negative voltage components. To mitigate this limitation, a low complexity and efficient signal shaper was developed, as described in [42]. Another approach is to rely on efficient charge recovery logic based AC computing, as described in [45].

The feasibility of the proposed approach has been demonstrated by recent results where the power consumed by a processing block (that relies on RF power and AC computing) has been reduced by more than an order of magnitude. Two examples that are implemented with AC computing include an 8-bit arithmetic logic unit to implement general purpose digital functions and a bit serialized SIMON32/64 (32-bit plaintext with a 64-bit key) core for lightweight encryption [44]. Both blocks operate with the harvested $13.56 \mathrm{MHz}$ carrier signal, which serves as both the power and clock signal. Both ALU and SIMON are also implemented with a conventional approach that relies on rectification and DC computing. The overall power consumption is compared in Fig. 6 for both blocks. For the 8-bit ALU, the proposed approach achieves up to $16.2 \mathrm{X}$ reduction in power whereas for the SIMON core, power is reduced by up to $34 \mathrm{X}$ and the energy efficiency $(\mathrm{kb} / \mathrm{sec} / \mu \mathrm{W})$ is enhanced by $27 \mathrm{X}$. The larger reduction for the SIMON core is primarily due to the existence of a large number of registers (required for bit serialized architecture) where AC computing is highly beneficial due to much smaller and efficient register implementation.

An important future work for AC computing is the development of a system-level energy management methodology. Since the modulation/demodulation and memory blocks as well as the supercapacitor require a DC voltage, the energy management module should determine how to split the harvested energy into DC and AC paths, depending upon the incoming data, required data processing, and power consumption of each block. Another interesting direction would be to investigate on-chip AC energy storage methods such as a flywheel manufactured on-chip by utilizing MEMS technology. AC energy storage would eliminate rectification loss when storing energy that would be later used by the data processing block.

\section{APPLICATIONS}

\subsection{Smart Spaces - Beyond the IoT}

The proposed technology will make possible that all objects in our living-, working-, and traveling-spaces will be tagged with miniature passive RF-addressable tags, which can autonomously communicate and interact among themselves without the need for a centralized control. These interactions will enable 'smart spaces' wherein objects can collaboratively understand the 'space' around them and recognize dynamic activities and events therein. Such context-aware intelligent physical spaces will enable new levels of automated human-object interaction and control through efficient exchange of information and knowledge. The tags in this vision will be designed for seamless and ubiquitous deployment, with low cost, and with size and form factor which will allow them to blend with all objects in our everyday spaces; from furniture to smaller objects such as clothes, wallets or keys. This vision goes beyond today's IoT (Internet of Things), where IoT concept is limited to only powerful devices - typically possessing an embedded processor and traditional network communication, such as thermostats, cameras or light bulbs.

\subsection{Floating Implantable Microsystems}

The proposed research can be highly beneficial for brain implantable microsystems. The number of applications for implantable systems has been growing in the recent years with significant impact on disease treatment and general healthcare. In the design of implantable devices, providing wireless power to the device and data communication link are critical parts of the system design. Power harvesting and telemetry through inductive coupling present a commonly used solution. As the bandwidth of the communication link is limited, raw sensor data need to be processed and compressed before transmission. Since the power is highly limited, performing these computational tasks requires extremely efficient computational techniques. Furthermore, the ability of the multiple implanted devices to communicate via backscattering exhibits significant opportunities in terms of real-time and closed-loop distributed sensing and decision making.

\subsection{Environmental and Structural Health Monitoring}

Wide deployment of wireless sensor nodes has been limited by the maintenance cost incurred by the battery replacement. Alternatively, proposed tags require only ambient RF signals to operate autonomously in these environments. In most of these applications, the detection of specific events (rather than continuous logging of sensor data) is critical. Unfortunately, detecting these critical events by processing raw sensor data increases the computational burden and therefore the overall energy cost. Proposed tags are able to carry out most of the processing locally and make decisions cooperatively with neighboring tags. We anticipate that the reduction in the energy cost for both computing and tag-to-tag communication achieved in this work will enable many of the potential applications that have been traditionally impeded.

\section{CONCLUSIONS}

Key enabling technologies have been described to develop RFpowered passive tags with wireless communication and computation capabilities. These tags represent the foundation for building intelligent tag networks, revitalizing the popular vision of smart 
dust. Critical enabling technologies include adaptive power management for backscatter based tag-to-tag communication and AC computing for energy-efficient and local decision making. The proposed framework is highly applicable to smart spaces and environmental/structural health monitoring where frequent battery changes are impractical, and brain implantable devices where a closed-loop system is essential for fast and efficient stimulation.

\section{REFERENCES}

[1] 2005. Class 1 Generation 2 UHF Air Interface Protocol Standard Version 1.0.9. EPCGlobal Inc.

[2] A. Athalye, J. Jian, Y. Karimi, S.R. Das, and Petar M. Djurić. 2016. Analog Front End Design for Tags in Backscatter-Based Tag-to-Tag Communication Networks. In IEEE Int. Symposium on Circuits and Systems (ISCAS).

[3] Akshay Athalye, Vladimir Savic, Miodrag Bolic, and Petar M Djuric. 2013. Novel semi-passive RFID system for indoor localization. Sensors fournal, IEEE 13, 2 (2013), 528-537.

[4] J. Blanckenstein, J. Klaue, and H. Karl. 2015. A Survey of Low-Power Transceivers and Their Applications. IEEE Circuits and Systems Magazine 15, 3 (2015), 6-17.

[5] Aniello Buonanno, Michele D'Urso, and Domenico Pavone. 2011. An ultra wideband system for RF Energy harvesting. In Antennas and Propagation (EUCAP), Proceedings of the 5th European Conference on. IEEE, 388-389.

[6] W. Che, Y. Yang, C. Xu, N. Yan, X. Tan, Q. Li, H. Min, and J. Tan. 2009. Analysis, design and implementation of semi-passive Gen2 tag. In IEEE International Conference on RFID. 15-19.

[7] H. Dagan, A. Shapira, A. Teman, A. Mordakhay, S. Jameson, E. Pikhay, V. Dayan, Y. Roizin, Eran E. Socher, and A. Fish. 2014. A Low-Power Low-Cost $24 \mathrm{GHz}$ RFID Tag With a C-Flash Based Embedded Memory. IEEE Journal of Solid-State Circuits 49, 9 (2014), 1942-1957.

[8] D. Daly and A. Chandrakasan. 2007. An energy-efficient OOK transceiver for wireless sensor networks. IEEE Journal of Solid-State Circuits 42, 5 (2007), 1003 1011

[9] Alanson P. Sample Daniel J. Yeager and Joshua R. Smith. 2008. RFID Handbook: Applications, Technology, Security, and Privacy. CRC Press, Chapter WISP: A Passively Powered UHF RFID Tag with Sensing and Computation.

[10] D. M. Dobkin. 2007. The RF in RFID: Passive UHF RFID in Practice. Elsevier Newnes.

[11] Klaus Finkenzeller. 2003. RFID Handbook: Fundamentals and Applications in Contactless Smart Cards and Identification. John Wiley \& Sons, Inc., New York, NY, USA.

[12] Xiongchuan Huang, Pieter Harpe, Guido Dolmans, Harmke de Groot, and John R Long. 2014. A 780-950 MHz, 64-146 $\mu \mathrm{W}$ power-scalable synchronized-switching ook receiver for wireless event-driven applications. IEEE Journal of Solid-State Circuits 49, 5 (2014), 1135-1147.

[13] J. M. Kahn, R. H. Katz, and K. S. J. Pister. 1999. Next Century Challenges: Mobile Networking for Smart Dust. In Proc. of the ACM/IEEE Int. Conf. on Mobile Computing and Networking. 271-278.

[14] Y. Karimi, A. Athalye, S. Das, P. Djurić, and M. Stanaćević. 2017. Design of Backscatter-Based Tag-to-Tag System. In IEEE Int. Conference on RFID (RFID).

[15] U. Karthaus and M. Fischer. 2003. Fully integrated passive UHF RFID transponder IC with $16.7-\mu \mathrm{W}$ minimum $\mathrm{RF}$ input power. IEEE fournal of Solid-State Circuits 38,10 (2003), 1602-1608.

[16] U. Karthaus and M. Fischer. 2003. Fully integrated passive UHF RFID transponder IC with $16.7 \mathrm{uW}$ minimum RF input power. IEEE Journal of Solid-State Circuits 38 10 (2003), $1602-1608$

[17] Bryce Kellogg, Aaron Parks, Shyamnath Gollakota, Joshua R. Smith, and David Wetherall. 2014. Wi-fi Backscatter: Internet Connectivity for RF-powered Devices. In Proceedings of the 2014 ACM Conference on SIGCOMM (SIGCOMM '14). ACM, New York, NY, USA, 607-618. https://doi.org/10.1145/2619239.2626319

[18] V. Kuhn, C. Lahuec, F. Seguin, and C. Person. 2015. A Multi-Band Stacked RF Energy Harvester With RF-to-DC Efficiency Up to 84\%. IEEE Transactions on Microwave Theory and Techniques 63, 5 (May 2015), 1768-1778.

[19] Y. Lee, D. Blaauw, and D. Sylvester. 2016. Ultralow Power Circuit Design for Wireless Sensor Nodes for Structural Health Monitoring. Proc. IEEE 104, 8 (Aug 2016), 1529-1546.

[20] S. Lin, J. Zhang, G. Zhou, L. Gu, J. Stankovic, and T. He. 2006. ATPC: adaptive transmission power control for wireless sensor networks. In Proc. of the Int. Conf. on Embedded Networked Sensor Systems. 223-236.

[21] Vincent Liu, Aaron Parks, Vamsi Talla, Shyamnath Gollakota, David Wetherall, and Joshua R. Smith. 2013. Ambient Backscatter: Wireless Communication Out of Thin Air. In Proc. ACM SIGCOMM.

[22] Xiao Lu, Ping Wang, Dusit Niyato, Dong In Kim, and Zhu Han. 2015. Wireless networks with RF energy harvesting: A contemporary survey. IEEE Communications Surveys \& Tutorials 17, 2 (2015), 757-789.
[23] Y. Lu, H. Dai, M. Huang, M. K. Law, S. W. Sin, S. P. U, and R. P. Martins. 2017. A Wide Input Range Dual-Path CMOS Rectifier for RF Energy Harvesting. IEEE Transactions on Circuits and Systems II: Express Briefs 64, 2 (Feb 2017), 166-170.

[24] Y. S. Luo and S. I. Liu. 2017. A Voltage Multiplier With Adaptive Threshold Voltage Compensation. IEEE Journal of Solid-State Circuits 52, 8 (Aug 2017), 2208-2214.

[25] Clemens Moser, Lothar Thiele, Davide Brunelli, and Luca Benini. 2007. Adaptive power management in energy harvesting systems. In Proceedings of the conference on Design, automation and test in Europe. EDA Consortium, 773-778.

[26] P.V. Nikitin, S. Ramamurthy, R. Martinez, and K.V.S. Rao. 2012. Passive tag-to-tag communication. In RFID (RFID), 2012 IEEE International Conference on. 177 -184.

[27] Emil Nilsson and Christer Svensson. 2014. Power consumption of integrated low-power receivers. IEEE Journal on Emerging and Selected Topics in Circuits and Systems 4, 3 (2014), 273-283.

[28] V.G. Oklobdzija, D. Maksimovic, and Fengcheng Lin. 1997. Pass-transistor adiabatic logic using single power-clock supply. IEEE Transactions on Circuits and Systems II: Analog and Digital Signal Processing 44, 10 (1997), 842-846.

[29] Chulsung Park and Pai H Chou. 2006. Ambimax: Autonomous energy harvesting platform for multi-supply wireless sensor nodes. In Sensor and Ad Hoc Communications and Networks, 2006. SECON'06. 2006 3rd Annual IEEE Communications Society on, Vol. 1. IEEE, 168-177.

[30] V. Pillai, H. Heinrich, D. Dieska, P.V. Nikitin, R. Martinez, and K.V.S. Rao. 2007. An ultra-low-power long range battery/passive RFID tag for UHF and microwave bands with a current consumption of $700 \mathrm{nA}$ at $1.5 \mathrm{~V}$. IEEE Transactions on Circuits and Systems I: Regular Papers 54, 7 (2007), 1500-1512.

[31] Nathan M Pletcher, Simone Gambini, and Jan Rabaey. 2009. A $52 m u$ W Wake-Up Receiver With $-72 \mathrm{dBm}$ Sensitivity Using an Uncertain-IF Architecture. IEEE fournal of solid-state circuits 44, 1 (2009), 269-280.

[32] B. Ransford, S. Clark, M. Salajegheh, and K. Fu. 2008. Getting things done on computational RFIDs with energy-aware checkpointing and voltage-aware scheduling. In Proceedings of USENIX Workshop on Power Aware Computing and Systems (HotPower).

[33] Srinivas S. Reddy and C.R. Murthy. 2012. Dual-stage power management algorithms for energy harvesting sensors. IEEE Transactions on Wireless Communications 11, 4 (2012), 1434-1445.

[34] S. Younis and T. Knight. 1994. Asymptotically zero energy computing using splitlevel charge recovery logic. Technical Report AITR-1500, MIT AI Laboratory. (June 1994).

[35] Z. Safarian and H. Hashemi. 2014. Wirelessly Powered Passive Systems With Dynamic Energy Storage Mechanism. IEEE Transactions on Microwave Theory and Techniques 62, 4 (2014), 1012-1021.

[36] Z. Shen, A. Athalye, and P. M. DjuriÄĞ. 2016. Phase Cancellation in BackscatterBased Tag-to-Tag Communication Systems. IEEE Internet of Things fournal 3, 6 (Dec 2016), 959-970. https://doi.org/10.1109/JIOT.2016.2533398

[37] Farhan Simjee and Pai H Chou. 2006. Everlast: long-life, supercapacitor-operated wireless sensor node. In Low Power Electronics and Design, 2006. ISLPED'06. Proceedings of the 2006 International Symposium on. IEEE, 197-202.

[38] C. Song, Y. Huang, P. Carter, J. Zhou, S. Yuan, Q. Xu, and M. Kod. 2016. A Novel Six-Band Dual CP Rectenna Using Improved Impedance Matching Technique for Ambient RF Energy Harvesting. IEEE Transactions on Antennas and Propagation 64, 7 (July 2016), 3160-3171.

[39] S. Sudevalayam and P. Kulkarni. 2011. Energy harvesting sensor nodes: Survey and implications. IEEE Communications Surveys \& Tutorials 13, 3 (2011), 443-461.

[40] N. Tran, B. Lee, and J-W. Lee. 2007. Development of long-range UHF-band RFID tag chip using Schottky diodes in standard CMOS technology. In IEEE Radio Frequency Integrated Circuits (RFIC) Symposium. 281-284.

[41] Christopher M Vigorito, Deepak Ganesan, and Andrew G Barto. 2007. Adaptive control of duty cycling in energy-harvesting wireless sensor networks. In Sensor, Mesh and Ad Hoc Communications and Networks, 2007. SECON'07. 4th Annual IEEE Communications Society Conference on. IEEE, 21-30.

[42] T. Wan, Y. Karimi, M. Stanaćević, and E. Salman. 2017. Energy efficient AC computing methodology for wirelessly powered IoT devices. In Circuits and Systems (ISCAS), 2017 IEEE International Symposium on. 1-4.

[43] T. Wan, Y. Karimi, M. Stanaćević, and E. Salman. 2017. Perspective Paper: Can AC Computing Be an Alternative for Wirelessly Powered IoT Devices? IEEE Embedded Systems Letters 9, 1 (2017), 13-16.

[44] T. Wan and E. Salman. 2018. Ultra Low Power SIMON Core for Lightweight Encryption. In Circuits and Systems (ISCAS), 2018 IEEE Int. Symp. on.

[45] T. Wan, M. Stanaćević, and E. Salman. 2016. A new circuit design framework for IoT devices: Charge-recycling with wireless power harvesting. In Circuits and Systems (ISCAS), 2016 IEEE International Symposium on. 2046-2049.

[46] S. G. Younis and T. F. Knight. 1995. Non-dissipative rail drivers for adiabatic circuits. In Proceedings of the Conference on Advanced Research in VLSI. 404-414.

[47] H. Zhang, J. Gummeson, B. Ransford, and K. Fu. 2011. Moo: A Batteryless Computational RFID and Sensing Platform. Technical Report UM-CS-2011-020. Department of Computer Science, University of Massachusetts Amherst, Amherst, MA. http://www.cs.umass.edu/publication/details.php?id=2114 